\title{
Postprandial blood glucose and insulin responses to pre-germinated brown rice in healthy subjects
}

\author{
Yukihiko Ito*, Aya Mizukuchi* ${ }^{*}$ Mitsuo Kise* , Hiromichi Aoto*, Shigeru Yamamoto ${ }^{\dagger}$, \\ Rie Yoshihara ${ }^{*}$, and Jyunichi Yokoyama \\ ${ }^{*}$ Central Research Laboratory, FANCL CORPORATION, Yokohama, Japan; ${ }^{\dagger}$ Department of Inter- \\ national Public Nutrition, Institute of Health Biosciences, The University of Tokushima Graduate \\ School, Tokushima, Japan; and "Department of Internal Medicine, Jikei University, School of Medicine, \\ Tokyo, Japan
}

\begin{abstract}
Effects of pre-germinated brown rice (PGBR) on postprandial blood glucose and insulin concentrations were compared with brown rice $(B R)$ and white rice $(W R)$ in two studies. In the first study, we investigated the time course of postprandial blood glucose and insulin concentrations after ingesting 25\% (W/V) glucose solution, PGBR, BR or WR in 19 healthy young subjects. In the second study, dose-dependent effect of PGBR on the time course of postprandial blood glucose concentrations was compared among 4 different mixtures of PGBR and WR in 13 healthy young subjects. They were solely PGBR, 2/3 PGBR (PGBR : WR= 2 : 1), 1/3 PGBR (PGBR : WR=1 : 2) and solely WR. Each sample was studied on a different day. The samples were selected randomly by the subjects. All the rice samples contained $50 \mathrm{~g}$ of available carbohydrates. The previous day the subjects ate the assigned dinner by $9: 00$ pm and then were allowed only water until the examination. The next morning, they ingested each test rice sample with $150 \mathrm{ml}$ of water in 5-10 min. Blood was collected into capillary tubes from finger at $0,30,60,90$ and $120 \mathrm{~min}$ after the ingestion. The incremental areas under the curve (IAUC) of blood glucose concentrations (IAUC-Glc) for $120 \mathrm{~min}$ after the administration of PGBR and BR were lower than those after WR. In contrast the IAUC-Glc of BR and PGBR were not different (Study 1). The higher the ratio of PGBR/WR, the lower the glycemic index became (Study 2). These results suggest that intake of PGBR instead of WR is effective for the control of postprandial blood glucose concentration without increasing the insulin secretion. J. Med. Invest. 52 : 159-164, August, 2005
\end{abstract}

Keywords : pre-germinated brown rice, brown rice, white rice, blood glucose, blood insulin, human

\section{INTRODUCTION}

About 150 million people in the world were living with diabetes in the year 2000, and the number is anticipated to increase to 220 million in 2015. In Asia,

Received for publication January 11, 2005 ; accepted February 16, 2005.

Address correspondence and reprint requests to Yukihiko Ito, Central Research Center, FANCL Corporation, 12-13 Kamishinano, Totsuka-ku, Yokohama 244-0806, Japan and Fax: +81-45-820-3509. the great increase is estimated in India, China and Japan (1).

Major risk factors of type 2 diabetes are genetics, age and obesity. The risk increases by life style such as smoking, physical activity and alcohol drinking (2-5). In the latest research, high postprandial blood glucose and insulin concentrations are the risk-factors of diabetes or cardiovascular disease $(6,7)$. The incidence of cardiac infarction in type 2 diabetes patients was shown to relate positively with blood glucose con- 
centration one hour after breakfast (8). A strong relationship was observed among a high carbohydrate diet, high blood glucose concentration and risk of cardiovascular diseases (9). DECODE (Diabetes Epidemiology : Collaborative analysis of Diabetic criteria in Europe) study involving 25,000 subjects showed that the high blood glucose concentration, as measured by oral glucose tolerance test (OGTT), increased the relative risk of mortality from large artery disease (10). These results suggest that high blood glucose concentration is a risk-factor for diabetes and its complications. These results indicate the importance of staple carbohydrate-rich foods which do not increase the postprandial blood glucose level quickly.

Jenkins, et al. $(11,12)$ proposed in 1981 glycemic index (GI) to indicate the level of postprandial blood glucose concentration after ingesting foods. White rice (WR), the most important staple food for Asians, has high GI due to the elimination of fiber-rich bran layer (13-15). Pre-germinated brown rice (PGBR) is a brown rice (BR) soaked in water for slight germination and is eaten in Japan. During the germination $\gamma$-aminobutyric acid (GABA) is produced, and therefore, PGBR is rich in GABA (16). PGBR also contains abundant dietary fiber, vitamins and minerals in the bran layer and germ. Previously, beneficial effects of PGBR on fasting blood glucose level in streptozotocininduced diabetic rats was reported (17). However, influence of difference between PGBR, BR, and WR on blood glucose and insulin levels in humans is unclear. In the present study, our purpose was to determine the difference of PGBR, BR and WR for the control of postprandial blood glucose and insulin levels in humans.

\section{SUBJECTS AND METHODS}

The study was divided into two parts. In the first study, 19 healthy subjects were included (7 women and 12 men). Their mean age was $29 \pm 1$ y (mean \pm SE, range 23-41) and mean body mass index was $21.4 \pm 0.8 \mathrm{~kg} / \mathrm{m}^{2}$ (mean $\pm \mathrm{SE}$, range 15.4-28.8). In the second study 13 healthy subjects were enrolled ( 8 women and 5 men) with the mean age of $28 \pm 1 \mathrm{y}$ (mean \pm SE, range 25-32) and the mean body mass index of $19.2 \pm 0.8 \mathrm{~kg} / \mathrm{m}^{2}$ (mean $\pm \mathrm{SE}$, range 15.4 25.6).

The subjects had fasting blood glucose level less than $110 \mathrm{mg} / \mathrm{dl}$ and did not have a report of abnormal blood glucose level by the regular annual medical examination within the last one year. All the subjects had given their informed consent before entering into the study and the protocol was approved by the Institutional Review Board, FANCL CORP., which conformed to the Helsinki Declaration.

The study included six test meals : $25 \%(\mathrm{~W} / \mathrm{V})$ glucose solution, WR, BR, PGBR (18) and two kinds of mixed WR with PGBR (1/3 PGBR and 2/3 PGBR; WR/PGBR ratios were $2: 1$ and $1: 2$, respectively). All the test samples contained $50 \mathrm{~g}$ of available carbohydrate. Characteristics of the test meals are given in Table 1. WR, BR and PGBR were the same type of short grain rice (Japonica) produced in the same area in Japan (Hokkaido). One test sample was given in a day for each subject. The order of the samples was decided randomly by the subjects. WR were cooked with $412 \mathrm{~g}$ of water, and 1/3 PGBR, 2/3 PGBR, and PGBR were cooked with $512 \mathrm{~g}$ of water by normal electric rice cooker (Mitsubishi Co. Ltd., Tokyo, Japan). On the other hand, induction heating rice cooker (Mitsubishi Co. Ltd., Tokyo, Japan) was used to cook

Table 1. Composition of test meals

\begin{tabular}{|c|c|c|c|c|c|c|}
\hline Test meal & $\begin{array}{l}\text { Serving } \\
\text { weight } \\
\text { (g) }\end{array}$ & $\begin{array}{c}\text { Available } \\
\text { carbohydrate } \\
\text { (g) }\end{array}$ & $\begin{array}{l}\text { Energy } \\
\text { (kcal) }\end{array}$ & $\begin{array}{l}\text { Protein } \\
\text { (g) }\end{array}$ & $\begin{array}{l}\text { Fat } \\
(\mathrm{g})\end{array}$ & $\begin{array}{c}\text { Dietary fiber } \\
\text { (g) }\end{array}$ \\
\hline $25 \%(w / v)$ glucose & 200 & 50 & & 0 & 0 & 0 \\
\hline WR & 161 & 49.3 & 221.5 & 4.5 & 0.7 & 0.4 \\
\hline 1/3 PGBR & 169 & 49.7 & 227.4 & 4.6 & 1.1 & 0.9 \\
\hline 2/3 PGBR & 177 & 50.2 & 233.3 & 4.7 & 1.6 & 1.4 \\
\hline PGBR & 185 & 50.6 & 239.2 & 4.8 & 2.0 & 1.9 \\
\hline $\mathrm{BR}$ & 178 & 49.4 & 233.1 & 4.8 & 1.9 & 2.0 \\
\hline
\end{tabular}

WR: white rice. $1 / 3$ PGBR : mixture of pre-germinated brown rice to WR (WR/PGBR ratio is $2: 1$ )

2/3 PGBR: mixture of pre-germinated brown rice to WR (WR/PGBR is $1: 2$ ). PGBR : pre-germinated brown rice.

$\mathrm{BR}$ : brown rice.

Values are calculated by the data of raw rice. 
$\mathrm{BR}$ after $563 \mathrm{~g}$ of water was added. The quantity of the water was determined by a preliminary study that gave the best taste. Hard physical activity and alcohol were not allowed during the study period. Exclusion criteria of the subjects were bad health condition, lack of sleep and menstruation for women. Previous night all the subjects were given the same dinner which was nutritionally defined and ate it by $9: 00 \mathrm{pm}$ and then only the water was allowed. In the next morning, they ingested each test food with $150 \mathrm{ml}$ of water in 5-10 min. Before and after that, at 0 and 30, 60, 90 and 120 min., blood was collected into capillary tubes from finger. The blood glucose concentration was measured with DexterZ II (Bayer Medical Co., Ltd., Leverkusen, Germany) soon after collection. In the first study, another blood sample was also sampled from the cubital fossa vein at the same time only from 15 participants who agreed to participate. The blood sample was centrifuged and serum was used for the analysis of insulin by enzyme immunoassay (by blood analysis company, SRL Inc, Tokyo, Japan).

\section{Statistical analysis}

Data were shown as mean \pm SE. The incremental areas under the glucose curve (IAUC-Glc) and insulin curve (IAUC-I) were calculated according to the method of Wolever ignoring values beneath basal level. Glycemic index (GI) was also calculated by the same method (19). In the first study, glucose and insulin data were analyzed by one-way analysis of variance (ANOVA), followed by Bonferroni multiple comparison test of means if the ANOVA indicated significant differences. In the first and second studies, the GIs were analyzed by the Student's t-test. Statistical analysis was done by Stat View 5.0 (SAS Institute, Inc., USA). The limit of significance was set at $p<0.05$ (2-tailed).

\section{RESULTS}

In the first study, all the rice samples were well accepted and consumed within $10 \mathrm{~min}$. Time courses of the blood glucose concentration after the ingestion of $25 \%$ (W/V) glucose solution, WR, BR and PGBR are shown in Figure 1. Table 2 shows the GIs. They were significantly lower for BR and PGBR than for WR $(\mathrm{p}<0.05)$. The concentrations reached the peak

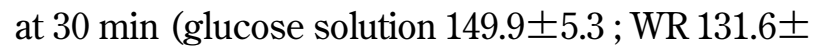
$4.1 ; \mathrm{BR} 130.9 \pm 4.1$ and PGBR $129.0 \pm 3.7 \mathrm{mg} / \mathrm{dl}$ ) and returned to the initial level at $120 \mathrm{~min}$ for all the samples. The values of $30 \mathrm{~min}$ were significantly lower

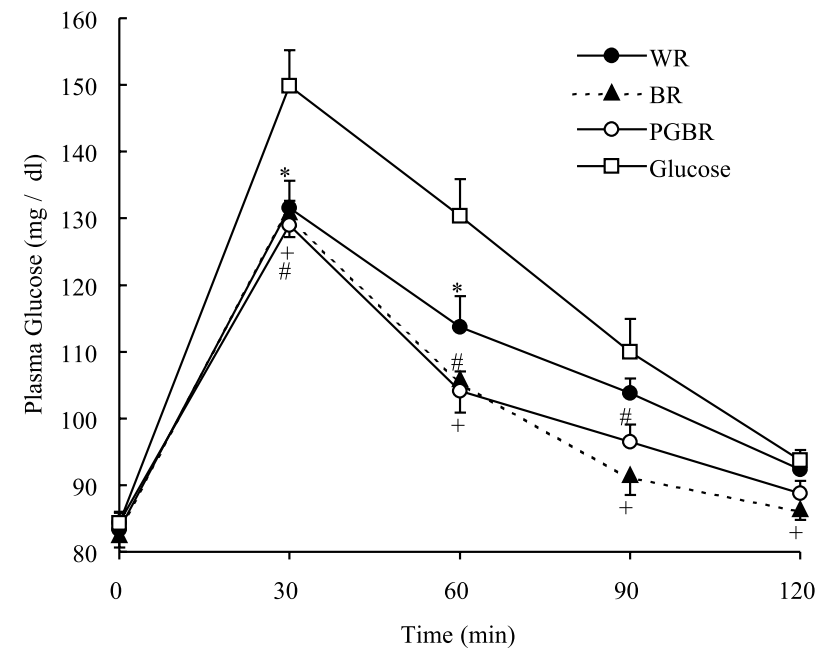

Figure 1. Postprandial blood glucose responses to each of the meals

WR: white rice. $\mathrm{BR}$ : brown rice. $\mathrm{PGBR}$ : pre-germinated brown rice. Each value represents the mean $\pm \mathrm{SE}$ of 19 subjects

*Values of WR group are significantly different from those of Glucose group $(\mathrm{p}<0.05)$

+Values of BR group are significantly different from those of Glucose group $(\mathrm{p}<0.05)$

\#Values of PGBR group are significantly different from those of Glucose group $(\mathrm{p}<0.05)$

Table 2. Glycemic Index of test meals (Study 1)

\begin{tabular}{cc}
\hline & GI (Glucose $=100)$ \\
\hline WR & $75.9 \pm 6.6$ \\
BR & $61.5 \pm 4.7^{*}$ \\
PGBR & $56.9 \pm 2.9^{*}$ \\
\hline
\end{tabular}

$\mathrm{WR}$ : white rice, $\mathrm{BR}$ : brown rice, $\mathrm{PGBR}$ : pre-germinated brown rice. Each value represents the mean \pm SE

* Significant difference compared to WR of Study 1 by Student's t-test ; $<<0.05(n=19)$

for the WR, BR and PGBR than for the glucose group $(p<0.05)$. The IAUC-Glc and IAUC-I for the first 60 min and for the entire 120 min of the study are shown in Figure 2. There was no significant difference among the IAUC-Glc of all the rice samples for the first 60 min (WR 3109 \pm 326.1 ; BR 2484.8 \pm 233.9 and PGBR $2354.2 \pm 189.9 \mathrm{mg} \cdot \mathrm{min} / \mathrm{dl})$. However, IAUC-Glc during the $120 \mathrm{~min}$ were significantly lower for the PGBR $(2354.2 \pm 189.9 \mathrm{mg} \cdot \mathrm{min} / \mathrm{dl})$ and the BR $(2484.8 \pm 233.9$ $\mathrm{mg} \cdot \mathrm{min} / \mathrm{dl})$ than for WR $(3109 \pm 326.1 \mathrm{mg} \cdot \mathrm{min} / \mathrm{dl})$. In contrast, the IAUC-Glc of BR and PGBR were not significantly different. IAUC-I for the entire $120 \mathrm{~min}$

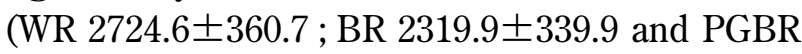
$2304.6 \pm 294.8 \mu \mathrm{U} \cdot \mathrm{min} / \mathrm{ml}$ ) was not significantly different among all the food samples.

Figure 3 shows the time courses of blood glucose concentrations after ingesting $25 \%$ (W/V) glucose solution, WR, 1/3 PGBR, 2/3 PGBR and PGBR in the 

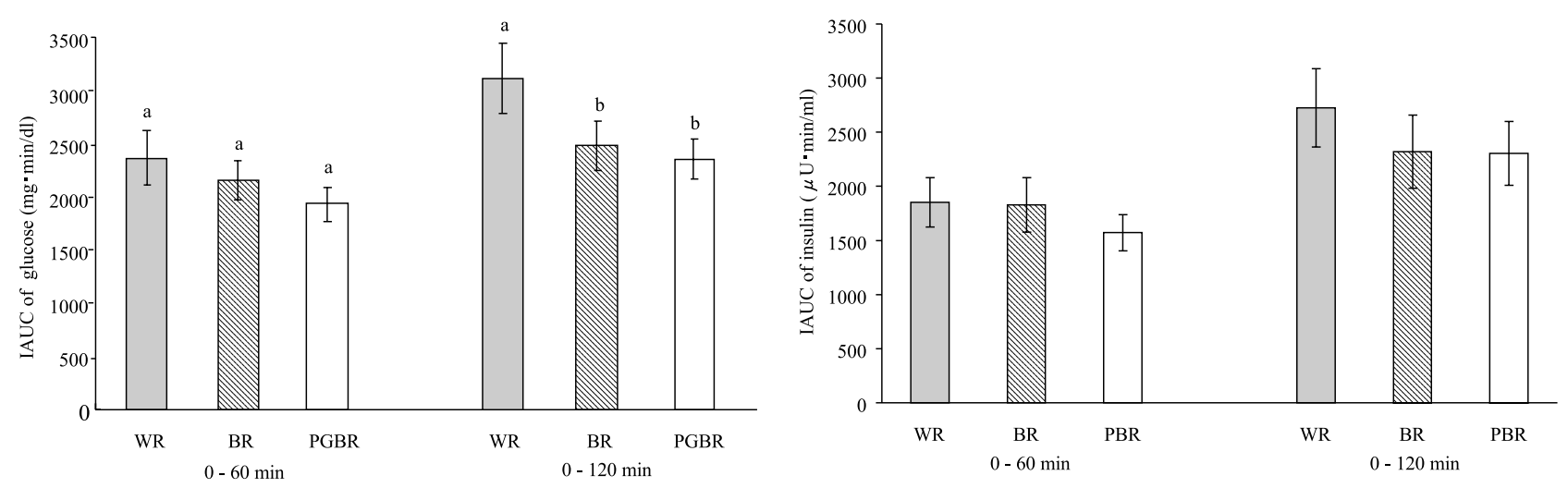

Figure 2. Incremental areas under the glucose and serum insulin corves to each of the meals WR: white rice. BR : brown rice. PGBR : pre-germinated brown rice. IAUC of the glucose value represents the mean $\pm \mathrm{SE}$ of 19 subjects. IAUC of the insulin value represents the mean $\pm \mathrm{SE}$ of 15 subjects.

a, b IAUC values of glucose concentration not sharing a common letter are significantly different $(p<0.05$ by Bonferroni multiple comparisons test ).

IAUC values of the insulin concentration are not significantly different among the 3 groupst $(p<0.05$ by Bonferroni multiple comparisons test).

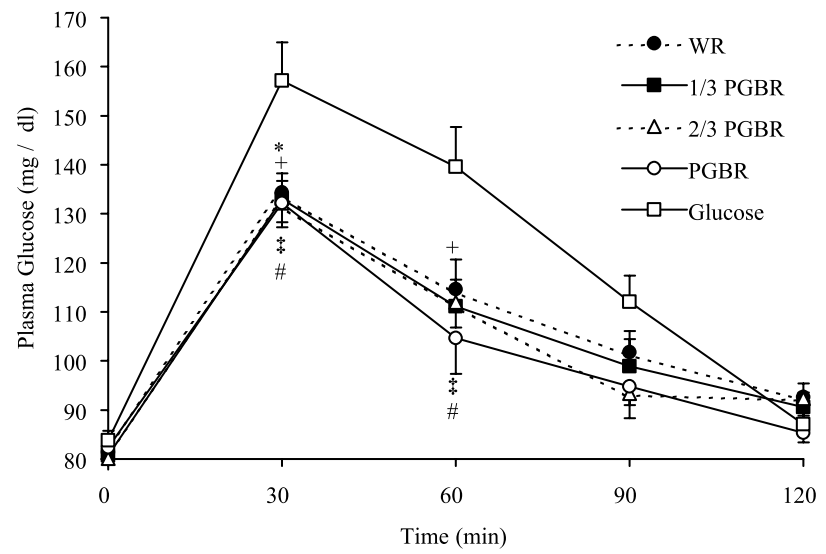

Figure 3. The influence of pre-germinated brown rice mixture on postprandial blood glucose responses

WR: white rice. $1 / 3$ PGBR: mixture of pre-germinated brown rice to $\mathrm{WR}$ (WR/PGBR ratio is $2: 1$ )

2/3 PGBR: mixture of pre-germinated brown rice to WR (WR/ PGBR ratio is $1: 2$ ). PGBR : pre-germinated brown rice.

Each value represents the mean $\pm S E$ of 13 subjects

*Values of WR group are significantly different from those of Glucose group $(\mathrm{p}<0.05)$

+Values of 1/3 PGBR group are significantly different from those of Glucose group $(\mathrm{p}<0.05)$

* Values of $2 / 3$ PGBR group are significantly different from those of Glucose group $(\mathrm{p}<0.05)$

\#Values of PGBR group are significantly different from those of Glucose group $(\mathrm{p}<0.05)$

second study. The blood glucose concentrations were significantly higher for the glucose than all the rice samples at 30 and $60 \mathrm{~min}(\mathrm{p}<0.05)$. The GIs are shown in Table 3. The GIs of all the rices were lower than that of glucose solution. The GIs of PGBR (55.2 \pm 2.9 and $54.4 \pm 5.1$ ) were significantly lower than those of WR (73.0 \pm 6.6 and $74.6 \pm 6.2$ ). However, there was no significant difference among the WR $(74.6 \pm 6.2)$ and

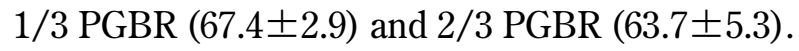

Table 3. Glycemic Index of test meals (Study 2)

\begin{tabular}{cc}
\hline & GI (Glucose $=100)$ \\
\hline WR & $74.6 \pm 6.2$ \\
$1 / 3$ PGBR & $67.4 \pm 2.9$ \\
$2 / 3$ PGBR & $63.7 \pm 5.3$ \\
PGBR & $54.4 \pm 5.1^{\#}$ \\
\hline
\end{tabular}

$\mathrm{WR}$ : white rice, $1 / 3 \mathrm{PGBR}$ : ratio of $\mathrm{PGBR}$ and $\mathrm{WR}=1: 2$,

1/2 PGBR : ratio of PGBR and WR=1 : 2, PGBR : pre-germinated brown rice.

Each value represents the mean $\pm \mathrm{SE}$

" $\mathrm{p}$ significant difference compared to WR of Study 2 by Student's t-test ; $<<0.05(n=13)$

\section{DISCUSSION}

IAUC-Glc during 120 min was significantly lower for BR and PGBR than for WR in healthy individuals in the first study and the GI decreased by the increase of PGBR/WR ratio in the second study. These results suggest that PGBR and BR are better than WR to prevent the rapid increase of postprandial blood glucose concentration without increasing the insulin secretion. The mechanism of it may be the higher dietary fiber concentration of BR and PGBR than WR. The concentrations of dietary fiber in BR, PGBR and WR were 2.0, 1.9 and $0.4 \mathrm{~g} / 100 \mathrm{~g}$ (Table 1). The fiber in the intestine inhibits the $\alpha$-amylase activity, the enzyme to digest starch, by rapping it. The fiber also raps the substrates (starch) in the small intestine. Our present finding and its postulated mechanism may be supported by the reports from the other researchers. There are a few studies which compared the postprandial blood glucose and/or insulin concentrations after taking BR 
and WR. O'Dea, et al. (14) observed that the glucose and insulin responses to BR tended to be smaller than those to WR, and that BR was hydrolyzed more slowly than WR in vitro (20). Hagiwara, et al. (17) reported that the blood glucose level and blood type-1 plasminogen activator inhibitor (PAI-1), which has been shown to be a major risk factor for cardiovascular disease in diabetes mellitus, significantly decreased in streptozotocin-induced diabetic rats by taking PGBR for 7 weeks. There are many reports which show the favorable effect of dietary fiber on the blood glucose concentration (21-25). A cohort study of 6 years also showed that foods rich in cereal fiber lower the risk of diabetes (23). Whole grains rich in fiber have beneficial effects of lowering fasting insulin level and decreasing the risk of cardiac disease in diabetes and obese adults $(24,25)$. From our results, together with the data of others, it could be concluded that BR and PGBR are good foods to prevent diabetes.

Some of our results were not consistent with the expected results. For instance, the time course of serum insulin concentration was not different significantly among the BR, PGBR and WR. IAUC-Glc for the first $60 \mathrm{~min}$ of the 3 samples were not significantly different, either. Such unexpected results may be due to small quantity of the test rice samples. In the previous studies in which subjects were given BR and WR, postprandial glucose concentrations at $30 \mathrm{~min}$ were similar when the starch was $50 \mathrm{~g}$ (26) but tended to be different when it was $75 \mathrm{~g}$ (14).

PGBR contains more GABA as compared with WR and BR (the contents of GABA in raw PGBR, BR and WR were $15 \mathrm{mg} / 100 \mathrm{~g}, 7 \mathrm{mg} / 100 \mathrm{~g}$ and $3 \mathrm{mg} / 100 \mathrm{~g}$, respectively). GABA is reported to stimulate the pancreatic beta cell and insulin secretion from pancreas (27). It was also reported that, when streptozotocininduced diabetic rats were injected with GABA agonist, their insulin levels increased and the blood glucose levels decreased (28). In this study, although PGBR was richer in GABA than BR, postprandial blood glucose and insulin responses of PGBR were similar to those of BR. Such results also indicate that GABA was not the factor to decrease the postprandial blood glucose concentration when the subjects took PGBR. However, we can not conclude from this study that GABA is not effective to control insulin secretion by the same reason that the quantity of the rice samples in this study was little.

The results of the present study suggest that the highly polished WR which is commonly used in Asian countries may easily increase the blood glucose concentration and the risk of diabetes. Asian people prefer
WR to BR because WR is softer and tastier than the BR. However, PGBR has less problems of BR by maintaining the benefits of BR. Therefore, PGBR may be recommended for more use considering its benefits.

\section{ACKNOWLEDGEMENTS}

The authors thank Naoko Fujimura and Hitomi Takano for expert technical assistance.

\section{REFERENCES}

1. Zimmet P, Alberti KG, Shaw J : Global and societal implications of the diabetes epidemic. Nature 414 : 782-787, 2001

2. Chan JM, Rimm EB, Colditz GA, Stampfer MJ, Willett WC : Obesity, fat distribution, and weight gain as risk factors for clinical diabetes in men. Diabetes Care 17 : 961-969, 1994

3. Manson JE, Rimm EB, Stampfer MJ, Colditz GA, Willett WC, Krolewski AS, Rosner B, Hennekens $\mathrm{CH}$, Speizer FE:Physical activity and incidence of non-insulin-dependent diabetes mellitus in women. Lancet 338 : 774-778, 1991

4. Jarrett RJ : Epidemiology and public health aspects of non-insulin-dependent diabetes mellitus. Epidemiol Rev 11 : 151-171, 1989

5. Barrett-Connor E: Epidemiology, obesity, and non-insulin-dependent diabetes mellitus. Epidemiol Rev $11:$ 172-181, 1989

6. Salmeron J, Ascherio A, Rimm EB, Colditz GA, Spiegelman D, Jenkins DJ, Stampfer MJ, Wing AL, Willett WC : Dietary fiber, glycemic load, and risk of NIDDM in men. Diabetes Care 20 : 545550, 1997

7. Leeds AR: Glycemic index and heart disease. Am J Clin Nutr 76 : 286S-289S, 2002

8. Hanefeld M, Fischer S, Julius U, Schulze J, Schwanebeck U, Schmechel H, Ziegelasch HJ, Lindner J : Risk factors for myocardial infarction and death in newly detected NIDDM : the Diabetes Intervention Study, 11-year follow-up. Diabetologia 39 : 1577-1583, 1996

9. Amano Y, Kawakubo K, Lee JS, Tang AC, Sugiyama M, Mori K : Correlation between dietary glycemic index and cardiovascular disease risk factors among Japanese women. Eur J Clin Nutr $58: 1472-1478,2004$

10. The DECODE study group. European Diabetes Epidemiology Group : Glucose tolerance and 
mortality : comparison of WHO and American Diabetes Association diagnostic criteria. Lancet 354 : 617-621, 1999

11. Jenkins DJ, Wolever TM, Taylor RH, Barker H, Fielden H, Baldwin JM, Bowling AC, Newman HC, Jenkins AL, Goff DV : Glycemic index of foods : a physiological basis for carbohydrate exchange. Am J Clin Nutr 34 : 362-366, 1981

12. Foster-Powell K, Holt SH, Brand-Miller JC : International table of glycemic index and glycemic load values. Am J Clin Nutr 76 : 5-56, 2002

13. Holt SH, Miller JC, Petocz P: An insulin index of foods : the insulin demand generated by $1000-$ $\mathrm{kJ}$ portions of common foods. Am J Clin Nutr 66 : 1264-1276, 1997

14. O'Dea K, Nestel PJ, Antonoff L: Physical factors influencing postprandial glucose and insulin responses to starch. Am J Clin Nutr 33:760-765, 1980

15. Ayuo PO, Ettyang GA : Glycaemic responses after ingestion of some local foods by non-insulin dependent diabetic subjects. East Afr Med J 73 : 782-5, 1996

16. Saikusa T, Horino T, Mori Y : Distribution of free amino acids in the rice kernel and kernel fractions and the effect of water soaking on the distribution. J Agric Food Chem 42:1122-1125, 1994

17. Hagiwara H, Seki T, Ariga T:The effect of pregerminated brown rice intake on blood glucose and PAI-1 levels in streptozotocin-induced diabetic rats. Biosci Biotechnol Biochem 68 : 444447, 2004

18. Aoto H, Sugino T, Shinmura H, Mizukuchi A, Kise M, Teramoto S, Someya S, Tsuchiya K, Ishiwata K: Germinated brown rice, Patent No. P3423931, JP, Jul. 7, 2003

19. Wolever TM, Jenkins DJ : The use of glycemic index in predicting the blood glucose response to mixed meals. Am J Clin Nutr $43: 167-172,1986$

20. O'Dea K, Snow P, Nestel P : Rate of starch hydrolysis in vitro as a predictor of metabolic responses to complex carbohydrate in vitro. Am J Clin Nutr 34 : 1991-1993, 1981

21. Madar $Z$ : Effect of brown rice and soybean dietary fiber on the control of glucose and lipid metabolism in diabetic rats : Am J Clin Nutr 38: 38893, 1983

22. Montonen J, Knekt P, Jarvinen R, Aromaa A, Reunanen A : Whole-grain and fiber intake and the incidence of type 2 diabetes. Am J Clin Nutr 77 : 622-629, 2003

23. Salmeron J, Manson JE, Stampfer MJ, Colditz GA, Wing AL, Willett WC : Dietary fiber, glycemic load, and risk of non-insulin-dependent diabetes mellitus in women. JAMA 277 : 472-477, 1997

24. Pereira MA, Jacobs DR Jr, Pins JJ, Raatz SK, Gross MD, Slavin JL, Seaquist ER: Effect of whole grains on insulin sensitivity in overweight hyperinsulinemic adults. Am J Clin Nutr 75 : 848-55, 2002

25. Liu S : Intake of refined carbohydrates and whole grain foods in relation to risk of type 2 diabetes mellitus and coronary heart disease. J Am Coll Nutr 21 : 298-306, 2002

26. Noriega E, Bouix O, Brun JF, Mestres C, Gauthier J, Oresetti A : Glycaemic and insulinaemic responses to white, brown and germinated rices in healthy subjects. Diab Nutr Metab $6: 215$ 221, 1993

27. Adeghate E, Ponery AS : GABA in the endocrine pancreas : cellular localization and function in normal and diabetic rats. Tisuue Cell $34: 1-6$, 2002

28. Gomez R, Asnis N, Tannhauser SL, Barros HM : GABA agonists differentially modify blood glucose levels of diabetic rats. Jpn J Pharmacol 80: 327-331, 1999 